\title{
Aplicaciones de dispositivos móviles como estrategia de aprendizaje en estudiantes universitarios de enfermería. Una mirada desde la fenomenología crítica
}

\begin{abstract}
Mobile Device Applications as a Learning Strategy in Nursing University Students. A look from Critical Phenomenology
\end{abstract}

\begin{abstract}
Aplicações de dispositivos móveis como estratégia de aprendizagem em estudantes de enfermagem. Um olhar da fenomenologia crítica
\end{abstract}

\author{
Jesús Roberto Garay Núñez \\ Universidad Autónoma de Sinaloa, México \\ jrgarayn@hotmail.com \\ https://orcid.org/0000-0002-0868-1344
}

\section{Resumen}

En la llamada sociedad del conocimiento o de la información, las nuevas formas de conectividad en el ámbito educativo han propiciado que los estudiantes incorporen nuevas herramientas como las aplicaciones de dispositivos móviles en sus procesos de autogestión del conocimiento. La omnipresencia de los dispositivos móviles en los espacios escolares está innovando la praxis del currículo en enfermería tanto en el aula como en el hospital y en escenarios comunitarios. El objetivo de este estudio fue determinar la experiencia vivida de alumnos de una licenciatura en enfermería en Culiacán, Sinaloa, con el uso de las aplicaciones en dispositivos móviles como estrategia de aprendizaje. Para ello se recurrió al método fenomenológico, el cual analiza las realidades cuya naturaleza y estructura peculiar solo pueden ser captadas desde el marco de referencia interno del sujeto que las vive y experimenta, y a la entrevista a profundidad como técnica. Como parte de los resultados surgió la conceptualización de cuatro categorías: 1) 


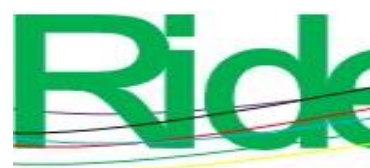

Revista Iberoamericana para la

Investigación y el Desarrollo Educativo

ISSN $2007-7467$

Aprendizaje centrado en las tecnologías de la información y la comunicación (TIC); 2) Aplicaciones móviles como estrategia de aprendizaje en la universidad; 3) Aplicaciones móviles como estrategia de aprendizaje en la práctica clínica, y 4) Aplicaciones móviles como estrategia de aprendizaje en la práctica comunitaria.

Palabras claves: aprendizaje móvil, enseñanza superior, recursos educativos abiertos.

\section{Abstract}

In the so-called knowledge or information society, new forms of connectivity in the educational field have led to the incorporation of new tools such as mobile device applications in the process of self-management of knowledge. The omnipresence of mobile devices in school spaces is innovating the praxis of the nursing curriculum in the classroom, hospital and community settings. The objective of this study was to determine the experience of students of a nursing degree in Culiacán, Sinaloa, with the use of mobile applications as a learning strategy. For this, the phenomenological method was used, which analyzes the realities whose peculiar nature and structure can only be captured from the internal frame of reference of the subject who lives and experiences them, and the in-depth interview as a technique. As part of the results, the conceptualization of four categories emerged: 1) Learning focused on information and communication technologies (ICT); 2) Mobile applications as a learning strategy in the university; 3) Mobile applications as a learning strategy in clinical practice, and 4) Mobile applications as a learning strategy in community practice.

Keywords: mobile learning, higher education, open educational resources.

\section{Resumo}

Na chamada sociedade do conhecimento ou da informação, novas formas de conectividade no campo educacional levaram os alunos a incorporar novas ferramentas, como aplicativos de dispositivos móveis, em seus processos de autogerenciamento do conhecimento. A onipresença de dispositivos móveis nos espaços escolares está inovando a práxis do currículo de enfermagem, tanto na sala de aula quanto no hospital e na comunidade. O objetivo deste estudo foi determinar a experiência de estudantes de um curso de enfermagem em Culiacán, Sinaloa, com o uso de aplicativos móveis como estratégia de aprendizado. Para isso, foi utilizado o método 


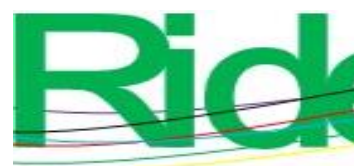

Revista Iberoamericana para la Investigación y el Desarrollo Educativo

ISSN $2007-7467$

fenomenológico, que analisa as realidades cuja natureza e estrutura peculiar só podem ser capturadas a partir do quadro de referência interno do sujeito que os vive e os experimenta, e da entrevista em profundidade como técnica. Como parte dos resultados, emergiu a conceitualização de quatro categorias: 1) Aprendizado voltado para as tecnologias da informação e comunicação (TIC); 2) Aplicações móveis como estratégia de aprendizagem na universidade; 3) Aplicativos móveis como estratégia de aprendizado na prática clínica e 4) Aplicativos móveis como estratégia de aprendizado na prática comunitária.

Palavras-chave: aprendizagem móvel, ensino superior, recursos educacionais abertos.

Fecha Aceptación: Diciembre 2019

\section{Introducción}

La introducción de las tecnologías de la información y la comunicación (TIC) en las aulas pone en evidencia la necesidad de una nueva definición de roles, especialmente para alumnos y docentes. Los primeros, gracias a estas nuevas herramientas, pueden adquirir mayor autonomía y responsabilidad en el proceso de aprendizaje, lo que obliga al docente a salir de su rol clásico como única fuente de conocimiento. Esto genera incertidumbres, tensiones y temores; realidad que obliga a una readecuación creativa de la institución escolar (Lugo, 2008).

Los sistemas escolares se ven enfrentados así a la necesidad de una transformación mayor e ineludible: evolucionar de una educación que servía a una sociedad industrial a otra que prepare a los educandos a desenvolverse en la sociedad del conocimiento. Los estudiantes deben estar preparados para desempeñarse en trabajos que aún hoy no existen y estar dispuestos a renovar continuamente una parte importante de sus conocimientos y habilidades; deben adquirir nuevas competencias coherentes con este nuevo orden: habilidades de manejo de información, comunicación, resolución de problemas, pensamiento crítico, creatividad, innovación, autonomía, colaboración y trabajo en equipo, entre otras (Binkley et al., 2010).

Esta actualización implica, en primer lugar, un desafío pedagógico para incorporar las TIC al aula y en el currículo escolar; la adecuación de la formación inicial y en servicio de los docentes, y políticas públicas que aseguren la implementación sistémica de reformas que impacten en los sistemas educativos de manera integral, lo que incluye asegurar la cobertura y calidad de la infraestructura tecnológica (hardware, software y acceso a servicios de 


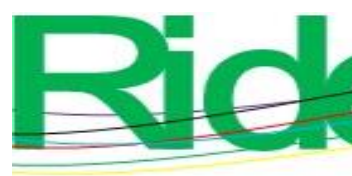

\section{Revista Iberoamericana para la Investigación y el Desarrollo Educativo ISSN $2007-7467$}

información y comunicación). Junto con esto, las TIC también presentan potenciales beneficios para mejorar la gestión escolar, lo que implica, también, preparar a directivos y administrativos en estas nuevas tecnologías (Organización de las Naciones Unidas para la Educación, la Ciencia y la Cultura [Unesco], 2013).

La escuela como espacio formal de educación con sus asignaturas, aulas, y espacios y tiempos de enseñanza y aprendizaje requiere ser transformado para ser más permeable y dinámico. La cultura de la sociedad del conocimiento obliga a tener la apertura necesaria para pensar de manera distinta la educación. Repensar la arquitectura de la escuela, el espacio de aprendizaje (que puede ser con distintas modalidades de virtualidad), el poder en la distribución del conocimiento. ¿Cómo podemos tener relaciones más horizontales entre quienes aprenden y los que educan en la escuela? ¿Cómo pueden nuestros sistemas educativos ser más abiertos con diversos actores sociales? ¿Cómo pueden enriquecerse nuestras formas de aprender y enseñar con la colaboración como dinámica de construcción social de conocimiento? (Cobo y Moravec, 2011).

Se espera que el aumento en los niveles de uso de los recursos TIC impulse no solo una transformación en los modelos pedagógicos, sino en el fomento de nuevos escenarios de adquisición de autonomía del proceso de enseñanza-aprendizaje en el aula. Además, si las tecnologías de información generan nuevos lenguajes y formas de representación, y facilitan la creación de nuevos escenarios de aprendizaje, las instituciones educativas no pueden permanecer al margen; deben conocer y utilizar estos nuevos lenguajes y formas de comunicación. Es urgente que las instituciones educativas se apropien de recursos, formen responsablemente a sus docentes en el uso de los nuevos medios y creen las condiciones para que sus alumnos se beneficien por igual de ellos y tengan igualdad de oportunidades en su acceso (Coll, Mauri y Onrubia, 2006).

En el ámbito educativo, las TIC proveen un sin número de herramientas, recursos, medios y formatos que posibilitan estrategias didácticas para facilitar la construcción de conocimientos; por ejemplo: aulas virtuales, blogs didácticos, evaluaciones online, aprendizaje móvil, realidad virtual, entornos virtuales 3D, entre otros. No obstante, su éxito depende de la capacidad para integrar la tecnología en el plan de estudios y crear experiencias de aprendizaje personalizado para cada alumno transformando el aula en un entorno de aprendizaje colaborativo (Briede, Leal, Mora y Pleguezuelos, 2015). 


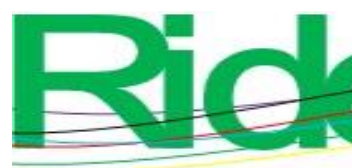

Revista Iberoamericana para la Investigación y el Desarrollo Educativo ISSN $2007-7467$

En este contexto, los estudiantes de pregrado de la licenciatura en enfermería no han sido ajenos a la gran influencia que ejercen los dispositivos móviles como herramienta interactiva de comunicación. Es decir, para la generación de los alumnos millennials es completamente común utilizar diferentes aplicaciones como estrategias de aprendizaje y forma de gestión del conocimiento para complementar los contenidos que se revisan en los espacios áulicos mediados por un facilitador.

La nueva forma de mediación interactiva a través de dispositivos móviles con conexión a internet permite a los estudiantes explorar a cualquier hora del día recursos ilimitados de nueva información que se genera casi al momento. Con aplicaciones de diversa temática de acuerdo con el área de interés o semestre de formación, de acuerdo con el plan de estudios de licenciatura en enfermería centrado en el aprendizaje y con un diseño de competencias profesionales integradas. Los estudiantes pueden acceder a recursos del área de fundamentos de enfermería, enfermería comunitaria, enfermería de la madre, de la recién nacida enfermería del niño y del adolescente, enfermería del adulto, enfermería del adulto mayor y enfermería en salud mental o a cualquier otra rama de la enfermería y en general de las ciencias de la salud.

\section{Material y método}

El método fenomenológico analiza las realidades cuya naturaleza y estructura peculiar solo pueden ser captadas desde el marco de referencia interno del sujeto que las vive y experimenta. En este caso, no se está estudiando una realidad “objetiva y externa”, igual para todos, sino una realidad cuya esencia depende del modo en que es vivida y percibida por el sujeto, una realidad interna y personal, única y propia de cada ser humano (Husserl, 1962).

La fenomenología y su método nacieron y se desarrollaron para estudiar estas realidades como son en sí, por lo cual se permite que estas se manifiesten por sí mismas sin constreñir su estructura desde afuera, sino respetándola en su totalidad. La fenomenología es el estudio de los fenómenos tal como son experimentados, vividos y percibidos por el hombre. Husserl acuñó el término (mundo de vida, mundo vivido) para expresar la matriz de este "mundo vivido, con su propio significado". Así, pues, en esta investigación de corte fenomenológico se realizarón las siguientes etapas: 1) Etapa previa: clarificación de los presupuestos; 2) Etapa descriptiva, y 3) Etapa estructural (Martínez, 2012, pág. 167-183). 


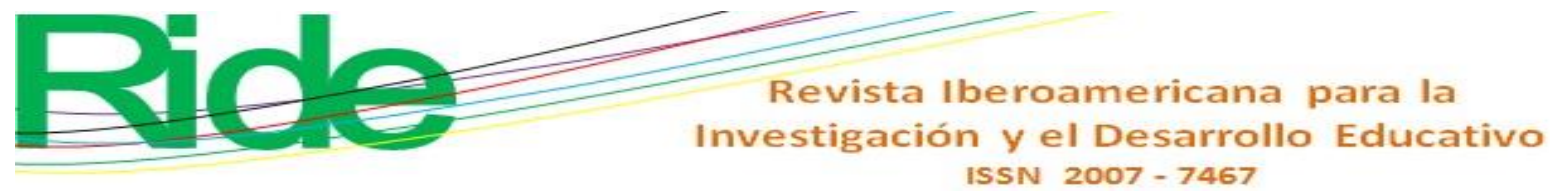

Sujetos de estudio

Para contactar a los entrevistados se utilizó el muestreo teórico en virtud de su disposición para suministrar información exhaustiva, entendido como las vivencias y experiencias investigativas como estudiantes. Se seleccionaron a dos estudiantes de sexo femenino y dos estudiantes de sexo masculino. Cada uno de los estudiantes pertenece a cada uno de los grados escolares en los que se divide la licenciatura en enfermería que cursaban en el momento de la entrevista. Cabe señalar que la investigación tuvo lugar en Culiacán, Sinaloa.

\section{Codificación, categorización y triangulación}

Se adoptaron, de manera integrativa y sistémica, las especificaciones de Strauss y Corbin (2002) para codificar, clasificar y categorizar toda la data generada por el estudio, específicamente las estrategias de codificación abierta (ubicación de conceptos y categorías similares en cuanto a propiedades y dimensiones), codificación axial (estructuración y relación entre categorías) y la codificación selectiva (integración de categorías en función de un concepto nuclear ordenador).

\section{Resultados}

Se presentan los resultados obtenidos en las entrevistas realizadas con el principio de saturación teórica relacionados con el tema en estudio, a través de la entrevista individual a profundidad con orientación fenomenológica, con el diálogo establecido entre el investigador y los estudiantes de enfermería en Culiacán, México. Después de realizar la recolección de los datos y el análisis de la información con los participantes durante la entrevista y a través del análisis de contenido, surgieron los significados de cuatro categorías teóricas (Ver tablas 1,2, 3 y 4). 


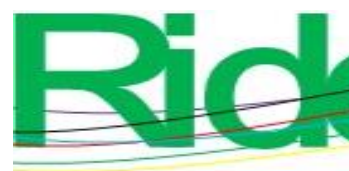

Revista Iberoamericana para la

Investigación y el Desarrollo Educativo ISSN $2007-7467$

Tabla 1. Categoría fenomenológica: Aprendizaje centrado en las TIC

\begin{tabular}{|c|c|}
\hline $\begin{array}{c}\text { Categoría } \\
\text { fenomenológica }\end{array}$ & 1. Aprendizaje centrado en las TIC \\
\hline $\begin{array}{l}\text { Subcategoría } \\
\text { (dimensión) }\end{array}$ & $\begin{array}{l}\text { El móvil como centro de la metacognición en el proceso de } \\
\text { aprendizaje }\end{array}$ \\
\hline Extractos discursivos & $\begin{array}{l}\text { 1. "[Respira] Para mí es común en clase entrar a internet para } \\
\text { investigar o una teoría un concepto que vimos en clase, para eso lo } \\
\text { utilizo principalmente" (estudiante } 2 \text { ). } \\
\text { 2. "Es mejor traer los libros electrónicos que ir a la biblioteca de la } \\
\text { escuela (...) [M1: cabeza]. Además, casi no encuentro lo que } \\
\text { necesito, mejor investigo en la Red y guardo lo que necesito de } \\
\text { acuerdo a la materia en que me toca hacer tarea [M2: Mano]" } \\
\text { (estudiante 4) } \\
\text { 3. "Cuando me toca exponer conecto el cañón a mi móvil y bajo la } \\
\text { exposición [M1: mano]. Es libre de virus, no tengo que llevar la } \\
\text { computadora a la escuela y lo mejor, no me expongo en el camión } \\
\text { trayendo la computadora porque a veces se pone muy inseguro y peor } \\
\text { cuando vamos a la comunidad a prácticas [M2: ambas manos en las } \\
\text { piernas]" (estudiante 1). } \\
\text { 4. "Tenemos un grupo de WhatsApp [M1: señala el móvil], en donde } \\
\text { estamos todo el grupo, por ahí nos pasamos el material para las } \\
\text { exposiciones, videos, libros electrónicos, documentos de Word o } \\
\text { PDF. No hay límites para la comunicación a menos que se llene la } \\
\text { memoria del móvil" (estudiante } 3 \text { ). } \\
\text { 5. "Nosotros en el equipo utilizamos el móvil para grabar un video } \\
\text { [M1: mano en la cabeza] que nos pidieron en la clase de desarrollo } \\
\text { humano, realizamos un sociodrama en video, después lo editamos y } \\
\text { lo pasamos a la memoria USB para presentarlo en clase" (estudiante } \\
\text { 1). }\end{array}$ \\
\hline Interpretación & $\begin{array}{l}\text { La utilización del móvil como estrategia de aprendizaje en la } \\
\text { sociedad del conocimiento es ya toda una realidad. No se puede } \\
\text { concebir la educación sin la mediación de las TIC y las tecnologías } \\
\text { del aprendizaje y del conocimiento. Los estudiantes del siglo XXI } \\
\text { utilizan las aplicaciones móviles para investigar y gestionar su } \\
\text { conocimiento con una rapidez inusitada. Además, cuentan con } \\
\text { información de fácil acceso, con buena y comprobada evidencia } \\
\text { científica, ya que muchas de las aplicaciones que utilizan son de } \\
\text { fuentes recomendadas por la propia Organización Mundial de la } \\
\text { Salud (OMS) y por universidades de reconocido prestigio científico. } \\
\text { Estamos frente a la generación } 2.0 \text {, cuyos integrantes gestionan } \\
\text { información de fuentes y recursos electrónicos como libros, audios, }\end{array}$ \\
\hline
\end{tabular}




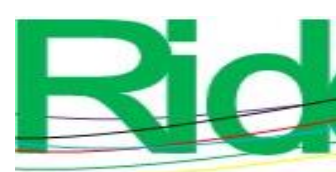

Revista Iberoamericana para la Investigación y el Desarrollo Educativo

ISSN $2007-7467$

\begin{tabular}{|l|l|}
\hline procedimientos y aplicaciones que lo mismo dan cuenta de \\
intervenciones clínicas que de intervenciones comunitarias. El \\
estudiante siempre está en la búsqueda de la mejor evidencia \\
científica y este ejercicio metacognitivo sin duda consolidará las \\
competencias que debe de adquirir en cada una de las unidades de \\
aprendizaje que está llevando a cabo en su formación como \\
licenciado en enfermería.
\end{tabular}

Fuente: Elaboración propia

Las TIC pueden ser entendidas como instrumentos psicológicos, utilizando los términos con los que Vigotsky acuñó el concepto, en tanto pueden llegar a ser herramientas que permiten pensar, sentir y actuar de forma individual o grupal. Esto, sin embargo, es solo un potencial, puede ser desplegado o no. Para que el potencial se convierta en una realidad se requiere que los usuarios, alumnos y maestros asignen deliberadamente una cierta funcionalidad a las herramientas. En otras palabras, depende de las prácticas educativas, del uso que se haga de las TIC, que estas se conviertan en verdaderos instrumentos de la mente (Coll et al., 2008). 


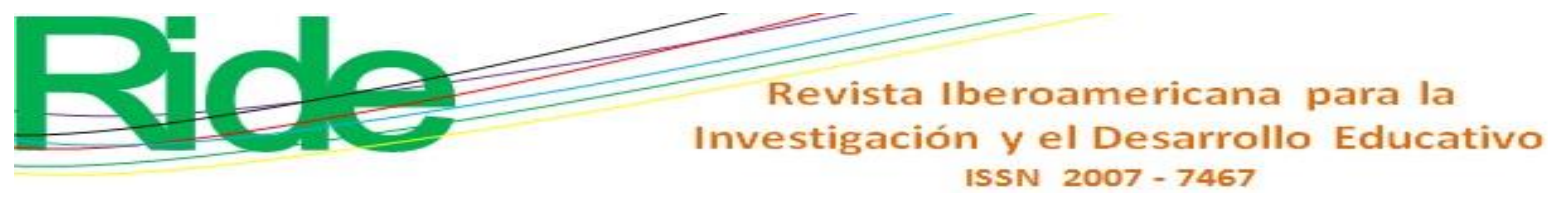

Tabla 2. Categoría fenomenológica: Aplicaciones móviles como estrategia de aprendizaje en la universidad

\begin{tabular}{|c|c|}
\hline $\begin{array}{c}\text { Categoría } \\
\text { fenomenológica }\end{array}$ & $\begin{array}{l}\text { 2. Aplicaciones móviles como estrategia de aprendizaje en la } \\
\text { universidad }\end{array}$ \\
\hline $\begin{array}{c}\text { Subcategoría } \\
\text { (dimensión) }\end{array}$ & 2.1. Utilización del móvil en clase \\
\hline $\begin{array}{l}\text { Extractos } \\
\text { discursivos }\end{array}$ & $\begin{array}{l}\text { 1. "Utilizo principalmente la aplicación del manual Merck. Es muy útil } \\
\text { para consultar cualquier área de la salud [M1: ambas manos]. Lo } \\
\text { mismo me ayuda en médico quirúrgica que en pediatría. Siempre lo } \\
\text { utilizo" (estudiante } 3 \text { ). } \\
\text { 2. "Un básico es el diccionario de términos médicos que es gratis en } \\
\text { Google Play. Sobre todo cuando tenemos clases de fisiopatología [M1: } \\
\text { mirada hacia abajo]. Que vienen muchos términos que son nuevos y } \\
\text { que no siempre se explica todo en clase" (estudiante 4). }\end{array}$ \\
\hline Interpretación & $\begin{array}{l}\text { La relación dialógica entre los docentes y los estudiantes también se ha } \\
\text { visto modificada por las TICS. Los alumnos optan en muchas } \\
\text { ocasiones por no realizar preguntas en clase, ya que muchas de sus } \\
\text { dudas las resuelven consultando las diferentes aplicaciones con las que } \\
\text { cuentan o en sitios de Internet. }\end{array}$ \\
\hline $\begin{array}{l}\text { Subcate } \\
\text { (dimen }\end{array}$ & 2.2. Toma de notas electrónicas \\
\hline $\begin{array}{l}\text { Extractos } \\
\text { discursivos }\end{array}$ & $\begin{array}{l}\text { 1. "Muchos de mis compañeros, contándome también yo [M1: mano } \\
\text { hacia el pecho], grabamos las clases que nos dan los maestros. Los } \\
\text { estamos grabando constantemente, es muy fácil y no ocupamos tomar } \\
\text { nota de nada" (estudiante 2) } \\
\text { 2. "Cuando están exponiendo en clase ya sea los compañeros o los } \\
\text { maestros, me gusta tomar fotos con el móvil de todas las diapositivas } \\
\text { que me parecen interesantes [M1: mirada hacia arriba]. Para repasar } \\
\text { después en la casa y aclarar dudas, sobre todo en el examen, ya que no } \\
\text { todos los maestros nos mandan el material y mejor tomo foto de todo" } \\
\text { (estudiante 3). }\end{array}$ \\
\hline Interpretación & $\begin{array}{l}\text { Toda la información está al alcance de un clic. La generación } 2.0 \text { ya no } \\
\text { toma notas ni escribe en libretas. Toma fotos de las diapositivas que se } \\
\text { analizan en clase, graba las clases de sus profesores, ya sea en } \\
\text { imágenes (video) o en audio, las comparten en grupos de WhatsApp o } \\
\text { a través de redes sociales. La comunicación es intersubjetiva mediada } \\
\text { por las TIC. } \\
\text { El estudiante ya no depende del facilitador para su aprendizaje, } \\
\text { depende de sus propias estrategias de gestión del conocimiento; utiliza } \\
\text { los dispositivos móviles, el internet y las aplicaciones de su interés } \\
\text { para gestionar competencias disciplinares y transversales para el } \\
\text { profesionista del siglo XXI. }\end{array}$ \\
\hline
\end{tabular}




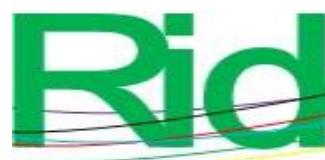

Revista Iberoamericana para la

Fuente: Elaboración propia

Se denomina aprendizaje móvil o m-learning al proceso que vincula el uso de dispositivos móviles con las prácticas de enseñanza-aprendizaje en un ambiente presencial o a distancia, el cual permite, por un lado, la personalización del aprendizaje conforme con los perfiles del estudiante, y por el otro, el acceso a contenidos y actividades educativas sin restricción de tiempo ni lugar. Mediante el aprendizaje móvil, se aprovecha la convergencia digital de los dispositivos móviles enfocando la capacidad de las aplicaciones que permiten registrar información de entornos reales, recuperar información disponible en Web y relacionar personas para realizar trabajo colaborativo (Romero, Molina y Chirino, 2010). 


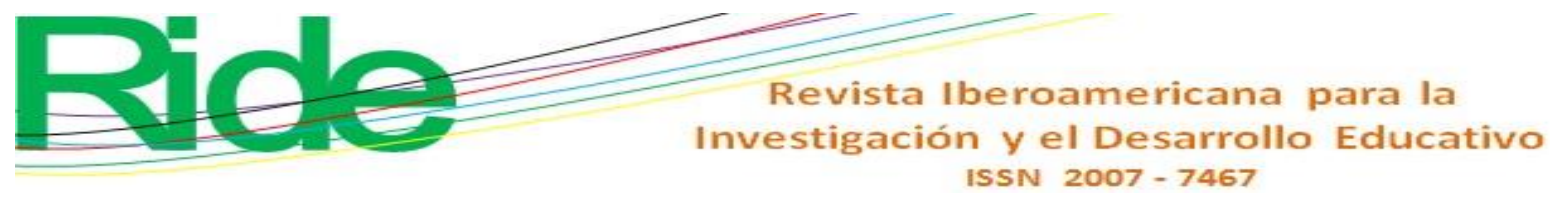

Tabla 3. Categoría fenomenológica: Aplicaciones móviles como estrategia de aprendizaje en la práctica clínica

\begin{tabular}{|c|c|}
\hline $\begin{array}{c}\text { Categoría } \\
\text { fenomenológica }\end{array}$ & $\begin{array}{l}\text { 3. Aplicaciones móviles como estrategia de aprendizaje en la práctica } \\
\text { clínica }\end{array}$ \\
\hline $\begin{array}{l}\text { Subcategoría } \\
\text { (dimensión) }\end{array}$ & El móvil centrado en la práctica clínica \\
\hline $\begin{array}{l}\text { Extractos } \\
\text { discursivos }\end{array}$ & $\begin{array}{l}\text { 1. "En la práctica clínica utilizo principalmente una aplicación que se } \\
\text { llama Enfermería Med IV. La utilizo principalmente para hacer } \\
\text { cálculos [M1: mirada hacia abajo] en la administración de } \\
\text { medicamentos y me olvido de la regla de tres. La verdad la } \\
\text { utilizamos la mayoría de los que estamos en prácticas. También sirve } \\
\text { para calcular [M2: mirada hacia abajo] las gotas por minutos de los } \\
\text { equipos de venoclisis" (estudiante 2). } \\
\text { 2. "La más importante para mí es NandaNocNic. Considero que es } \\
\text { una aplicación indispensable, ya que siempre nos están pidiendo } \\
\text { evidencias de proceso enfermero [M1: se toma de ambas manos] en } \\
\text { todos los servicios, al menos con un paciente [M2: señala el uno con } \\
\text { el dedo]. Es muy práctica y los diagnósticos enfermeros salen } \\
\text { automáticos; solo tú vas escogiendo cuál se adapta más de acuerdo a } \\
\text { los signos y síntomas que presenta cada paciente y los cuidados que } \\
\text { como enfermeros debemos de brindar. También tengo el libro de } \\
\text { Nanda, Noc y Nic en versión electrónica solo para complementar en } \\
\text { ocasiones lo que revisamos en práctica con la maestra" (estudiante } \\
\text { 4). } \\
\text { 3. "Utilizo casi siempre Elsevier Enfermería porque me explica paso } \\
\text { a paso los principales procedimientos y cuidados que debo aplicar a } \\
\text { los pacientes; sin embargo, claro, hay diferencias [M1: se cruza de } \\
\text { piernas] en los videos, las simulaciones de los laboratorios y la } \\
\text { realidad que vemos en los hospitales, pero sí he aprendido mucho } \\
\text { sobre todo reafirmando la práctica para tener más seguridad en el } \\
\text { hospital" (estudiante 2). }\end{array}$ \\
\hline Interpretación & $\begin{array}{l}\text { Las aplicaciones en el campo profesional de la enfermería han tenido } \\
\text { un desarrollo vertiginoso. Los estudiantes pueden acceder a un gran } \\
\text { catálogo de posibilidades para la gestión del conocimiento del campo } \\
\text { clínico en las diferentes áreas que comprende el currículo de } \\
\text { enfermería (fundamentos de enfermería, enfermería de la madre y el } \\
\text { recién nacido, enfermería del niño y del adolescente, enfermería del } \\
\text { adulto, enfermería del adulto mayor y enfermería en salud mental). } \\
\text { También se cuenta con aplicaciones móviles para la gestión del } \\
\text { cuidado basado en evidencia científica. La taxonomía nanda, noc y } \\
\text { nic es una de las más utilizadas no solo en aplicaciones móviles sino } \\
\text { también en libros electrónicos, en donde los alumnos pueden planear }\end{array}$ \\
\hline
\end{tabular}




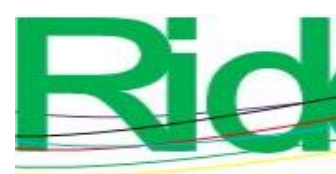

\begin{tabular}{|l|l|}
\hline y fundamentar el cuidado de enfermería centrado en la persona. \\
$\begin{array}{l}\text { Muchas de las aplicaciones que se utilizan son de gran calidad y } \\
\text { aceptación mundial. Por ejemplo, la app de Elsevier Enfermería } \\
\text { permite acceder a más de } 38 \text { 000 definiciones relacionadas con } \\
\text { enfermería y ciencias de la salud. Además, cuenta con alrededor de } \\
85 \text { habilidades y procedimientos dirigidos tanto a estudiantes como a } \\
\text { profesionistas. Y es completamente gratis. }\end{array}$ \\
$\begin{array}{l}\text { Las aplicaciones son de gran utilidad en los escenarios áulicos, de } \\
\text { simulación clínica y en los espacios hospitalarios para el aprendizaje } \\
\text { situado. }\end{array}$ \\
\hline
\end{tabular}

Fuente: Elaboración propia

El estudiante fabrica su propio conocimiento sobre nuevos pensamientos y conocimientos previos. Las aplicaciones móviles deben recomendar esquemas de virtualización de contextos y brindar mecanismos que posibiliten administrar dicho conocimiento, así como procedimientos de búsqueda de información (Soler, 2014). 


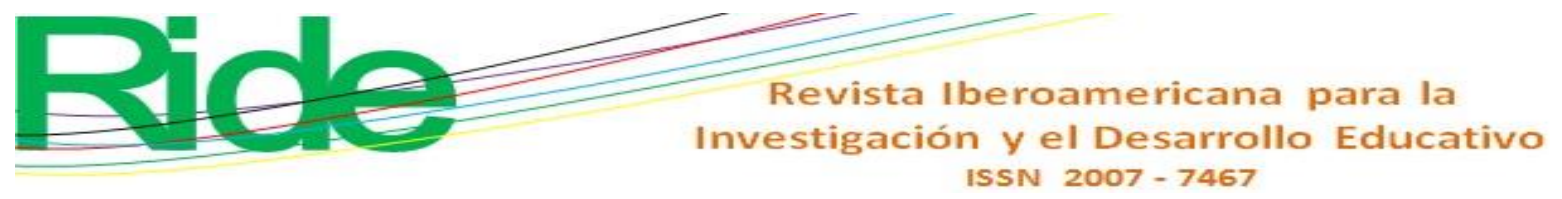

Tabla 4. Categoría fenomenológica: Aplicaciones móviles como estrategia de aprendizaje en la práctica comunitaria

\begin{tabular}{|c|c|}
\hline $\begin{array}{c}\text { Categoría } \\
\text { fenomenológica }\end{array}$ & $\begin{array}{l}\text { 4. Aplicaciones móviles como estrategia de aprendizaje en la práctica } \\
\text { comunitaria. }\end{array}$ \\
\hline $\begin{array}{l}\text { Subcategoría } \\
\text { (dimensión) }\end{array}$ & El móvil centrado en la práctica comunitaria \\
\hline $\begin{array}{l}\text { Extractos } \\
\text { discursivos }\end{array}$ & $\begin{array}{l}\text { 1. "En la práctica de enfermería de primer nivel utilicé una aplicación } \\
\text { que se llama VacunAPP [M1: cabeza]. Ahí viene muy bien explicado } \\
\text { el tipo de vacuna, y la dosis por grupos etarios. También vienen todas } \\
\text { las cartillas del sector salud. Además, te explica los mecanismos de } \\
\text { acción y que enfermedades previene. Ahh [M2: respira], también te } \\
\text { dice cuál es el centro de salud más cercano al sector en donde vives" } \\
\text { (estudiante 2). } \\
\text { 2. "Me ha funcionado muy bien ClikiSalud. Es una aplicación muy } \\
\text { recomendable por los manuales de promoción de la salud, que son } \\
\text { muy útiles sobre todo cuando tengo que preparar los temas para la } \\
\text { feria de salud [M1: se cruza de brazos] que organizamos en la } \\
\text { colonia. Se promueve el autocuidado y los estilos de vida saludables, } \\
\text { que son uno de los principales objetivos de la práctica comunitaria" } \\
\text { (estudiante } 3 \text { ). } \\
\text { 3. "La app Familia y Salud es muy recomendable, ya que puedes } \\
\text { bajar información de estilos de vida saludables, el plato del buen } \\
\text { comer, el vaso del buen beber, información de vacunas, información } \\
\text { de enfermedades, prevención de accidentes en casa [M1: respira]. } \\
\text { También cuenta con información de salud de acuerdo a la etapa de } \\
\text { vida de la persona y recomendaciones básicas de promoción de } \\
\text { salud" (estudiante } 4 \text { ). }\end{array}$ \\
\hline Interpretación & $\begin{array}{l}\text { La promoción de la salud y la prevención de la enfermedad es una } \\
\text { prioridad para la enfermería comunitaria. También en esta área se } \\
\text { cuenta con aplicaciones móviles que los profesionistas en formación } \\
\text { consultan para planear sus intervenciones educativas comunitarias, } \\
\text { teniendo como centro del cuidado y como célula básica de la } \\
\text { sociedad a la familia. } \\
\text { Los estilos de vida saludables, las detecciones oportunas de } \\
\text { prevención de enfermedades, la dieta, ejercicio y prácticas de } \\
\text { autocuidado son solo algunas de las temáticas con las que cuentan las } \\
\text { aplicaciones centradas en el área de enfermería comunitaria. } \\
\text { La atención de programas prioritarios de salud por grupos etarios, así } \\
\text { como el esquema de vacunación de niños, adolescentes, mujeres en } \\
\text { estado gestacional, adultos y adultos mayores. }\end{array}$ \\
\hline
\end{tabular}




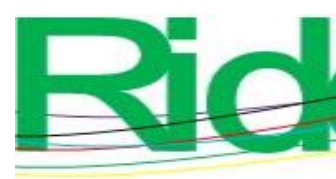

Revista Iberoamericana para la Investigación y el Desarrollo Educativo ISSN $2007-7467$

\begin{tabular}{|l|l|}
\hline Con todos estos elementos, los estudiantes están preparados para \\
desarrollar competencias profesionales a través del pensamiento \\
crítico y reflexivo, aunado a la integración de competencias \\
profesionales a través de la autogestión y mediación de la Web 2.0, \\
fundamentando el cuidado de la familia y la comunidad con \\
evidencia científica.
\end{tabular}

Fuente: Elaboración propia

Las plataformas virtuales debe garantizar un entorno adecuado de aprendizaje que permita la interactividad entre estudiantes y docentes y la gestión y organización del curso, los requerimientos necesarios de hardware y software, el número de usuarios, su compatibilidad con otras plataformas, la utilización de herramientas sincrónicas y asincrónicas, entre otras (Naranjo y Rivero, 2014).

\section{Discusión}

De acuerdo con Basantes, Naranjo, Gallegos y Benítez (2017), quienes realizaron un estudio sobre los dispositivos móviles en el proceso de aprendizaje de la Facultad de Educación Ciencia y Tecnología de la Universidad Técnica del Norte de Ecuador, la mayoría de estudiantes y docentes emplean los dispositivos móviles para comunicarse, para el entretenimiento y con un porcentaje ligero para la educación en orden descendente, lo que demuestra que se subutilizan los dispositivos móviles y que no se aprovechan los recursos que disponen para fortalecer el aprendizaje en la formación profesional o académica; por lo tanto, se debe capacitar a los docentes sobre el uso de los dispositivos móviles en la educación, con la finalidad de incorporar la tecnología móvil en su labor como una herramienta de apoyo para el desarrollo de sus clases, y generar con ello un ambiente de interacción, cooperación y colaboración.

En contraste con el caso anterior, en nuestro estudio se determinó que los estudiantes del pregrado en enfermería utilizan las aplicaciones en dispositivos móviles de forma constante en todos los espacios de formación profesional, es decir, en los espacios áulicos, en los espacios de simulación clínica, en los escenarios de prácticas clínicas de los diferentes hospitales del sector salud y en los espacios de la práctica comunitaria, teniendo como centro de actuación a la familia. 


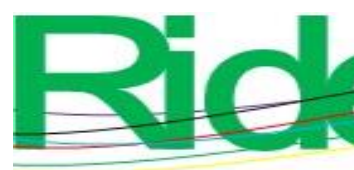

Revista Iberoamericana para la Investigación y el Desarrollo Educativo ISSN $2007-7467$

Los estudios e investigaciones llevadas a cabo en este ámbito indican que los proyectos y experiencias de innovación apoyados en el uso de tecnologías distintas a las convencionales se estrellan con los hábitos y la cultura tradicional del sistema escolar. Es inevitable que la incorporación de los materiales digitales supere múltiples resistencias y dificultades, y permita que en la educación los computadores sean de normal utilidad (Gros, 2000).

En otra investigación llevada a cabo por Muñoz (2010) se concluyó que los dispositivos móviles han demostrado ser útiles en la enseñanza médica. Los asistentes digitales personales han sido los dispositivos más estudiados en la literatura médica, aunque han ido desapareciendo del mercado y han sido sustituidos progresivamente por los smartphones. Aunque estos pueden realizar las funciones de los asistentes digitales personales y añaden una mayor capacidad de reproducir archivos multimedia y la posibilidad de conexión a redes inalámbricas, actualmente son dispositivos caros y requieren el contrato de una tarifa de datos para emplear toda su capacidad. Hay un creciente interés por los podcasts como instrumento para difundir conocimiento y es probable que en los próximos años esta tecnología adquiera más relevancia en la educación médica (Muñoz, 2010).

Ahora bien, aquí se encontró que en la educación en enfermería las aplicaciones móviles están cada vez más posicionadas. Uno de los principales recursos que utilizan los estudiantes de enfermería es la aplicación NandaNocNic como parte del proceso enfermero, el cual es la piedra angular para la gestión del cuidado de enfermería y determinar los diagnósticos para el mantenimiento o recuperación de la salud del individuo, familia o comunidad.

Como lo indica el informe de la Organización para la Cooperación y el Desarrollo Económicos [OCDE] (2003), existe una fuerte tensión entre los currículos tradicionales basados en contenidos bien definidos que el estudiantado debe aprender y saber reproducir, y el enfoque abierto que promueven las TIC. Los tipos y modos de estructuración del pensamiento de los sujetos que actúan con materiales digitales tendrán que ser necesariamente distintos de los que poseen los lectores habituales de documentos escritos. Es indudable que el empleo de estos nuevos recursos implicará una mayor integración de las instituciones educativas en el contexto de la sociedad de la información o era digital. Se trata de integrar las TIC al currículo, y de llevarlas a las aulas con sentido pedagógico (Iriarte, Said, Valencia y Ordoñez, 2015). 


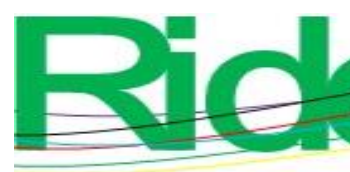

Revista Iberoamericana para la Investigación y el Desarrollo Educativo ISSN $2007-7467$

\section{Conclusiones}

No se puede concebir la educación del siglo XXI sin la presencia de las aplicaciones móviles como estrategia de autogestión del aprendizaje y metacognición de los estudiantes que utilizan en todos y cada una de los espacios de formación universitaria (aulas, laboratorio de simulación clínica, hospitales públicos y escenarios comunitarios). Sin duda, el pensamiento crítico y reflexivo es una competencia central en la formación de enfermeras y enfermeros en México.

Sin embargo, las aplicaciones móviles no sustituyen la relación de enseñanzaaprendizaje de los docentes con los estudiantes; hay que reconocer, sin embargo, que las TIC llegaron para innovar los espacios de formación universitaria y que los estudiantes cuentan con herramientas de aprendizaje y de conocimiento innovadoras y permanentemente actualizadas, con información de punta en todos los ámbitos y acentuaciones de la enfermería.

\section{Referencias}

Basantes, A. V., Naranjo, M. E., Gallegos, M. C. y Benítez, N. M. (2017). Los dispositivos móviles en el proceso de aprendizaje de la Facultad de Educación Ciencia y Tecnología de la Universidad Técnica del Norte de Ecuador. Formación universitaria, 10(2), 7988. https://dx.doi.org/10.4067/S0718-50062017000200009

Binkley, M., Erstad, O., Herman, J., Raizen, S., Ripley, M. and Rumble, M. (2010). Draft White Paper 1. Defining 21st century skills. London, England: Cisco, Intel and $\begin{array}{lll}\text { Microsoft. } & \text { Retrieved from }\end{array}$ https://oei.org.ar/ibertic/evaluacion/sites/default/files/biblioteca/24_defining-21stcentury-skills.pdf.

Briede, J.C., Leal, I.M., Mora, M. L., y Pleguezuelos, C.S. (2015). Propuesta de Modelo para el Proceso de Enseñanza Aprendizaje Colaborativo de la Observación en Diseño, Utilizando la Pizarra Digital Interactiva (PDI), Formación Universitaria, Recuperado dehttps://scielo.conicyt.cl/scielo.php?script=sci abstract\&pid=S071850062015000300003\&ln $\mathrm{g}=\mathrm{es} \& \mathrm{nrm}=\mathrm{iso}$

Cobo, C. y Moravec, J. W. (2011). Aprendizaje Invisible. Hacia una nueva ecología de la educación. Barcelona, España: Publicacions i Edicions de la Universitat de Barcelona. Recuperado de http://www.razonypalabra.org.mx/varia/AprendizajeInvisible.pdf. 


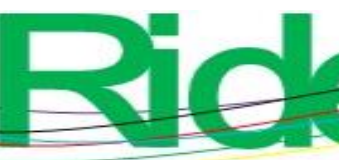

Revista Iberoamericana para la

Investigación y el Desarrollo Educativo

ISSN $2007-7467$

Coll, C., Mauri, T. y Onrubia, J. (2006). Análisis y resolución de casos-problema mediante el aprendizaje colaborativo. Revista de Universidad y Sociedad del Conocimiento, 3(2), $29-41$.

Recuperado

de

http://rusc.uoc.edu/rusc/ca/index.php/rusc/article/download/v3n2-coll-mauri-

onrubia/285-1203-2-PB.pdf.

Coll, C., Mauri, T. y Onrubia. J. (2008). La utilización de las tecnologías de la información y comunicación en la educación: del diseño técnico-pedagógico a las prácticas de uso. En Coll, C. y Monereo, C. (eds.), Psicología de la educación virtual (pp. 74-103). Madrid, España: Morata. Recuperado de https://mediacaotecnologica.files.wordpress.com/2012/08/psicologia-de-la-educacionvirtual-coll-y-monereo.pdf.

Fernández, A. y Rivero, M. (2014). Las plataformas de aprendizajes, una alternativa a tener en cuenta en el proceso de enseñanza aprendizaje. Revista Cubana de Informática Médica, 6(2), 207-221. Recuperado de http://scielo.sld.cu/scielo.php?script=sci arttext\&pid=S168418592014000200009\&lng =es\&tlng=es.

Gros, B. (2000). El ordenador invisible. Hacia la apropiación del ordenador en la enseñanza. Barcelona, España: Gedisa.

Husserl, E. (1962). Ideas relativas a una fenomenología pura y una filosofía fenomenológica. Ciudad de México, México: UNAM / FCE.

Iriarte, F., Said, E., Valencia, J. y Ordoñez, M. (2015). Propuesta de modelo para el fortalecimiento del uso de las TIC en contextos escolares. Bogotá D.C., Colombia: Universidad del Norte / Corporación Colombia Digital. Recuperado de https://www.calidadeducativasm.com/wpcontent/uploads/2015/10/Propuesta_modelo_fortalecimiento_uso_TIC_en_contextos_e scolares.pdf.

Lugo, M. T. (2010). Las políticas TIC en la educación de América Latina. Tendencias y experiencias. Revista Fuentes, 10, 52-68. Recuperado de http://www.memoria.fahce.unlp.edu.ar/art revistas/pr.7036/pr.7036.pdf.

Martínez M, M. (2012). Comportamiento humano: nuevos métodos de investigación (2. ${ }^{\mathrm{a}}$ ed.). México: Trillas. 


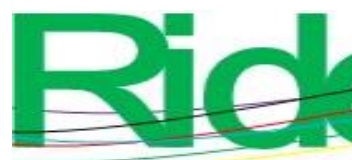

Revista Iberoamericana para la Investigación y el Desarrollo Educativo

ISSN $2007-7467$

Muñoz, C. F. (2010). Dispositivos móviles en la educación médica. Teoría de la Educación: Educación y Cultura en la Sociedad de la Información, 11(2), 28-45 Recuperado de https://www.redalyc.org/pdf/2010/201014893003.pdf.

Organización de las Naciones Unidas para la Educación, la Ciencia y la Cultura [Unesco]. (2013) Enfoques estratégicos sobre las Tics en educación en América latina y el caribe. Santiago, Chile: Organización de las Naciones Unidas para la Educación, la Ciencia y la Cultura. Recuperado de http://www.unesco.org/new/fileadmin/MULTIMEDIA/FIELD/Santiago/images/ticsesp.p df.

Organización para la Cooperación y el Desarrollo Económicos [OCDE]. (2003). Los desafíos de las Tecnologías de la Información y las Comunicaciones en la Educación. Madrid, España: Ministerio de Educación, Cultura y Deporte. Recuperado de https://www.oecdilibrary.org/education/los-desafios-de-las-tecnologias-de-la-informacion-y-lascomunicaciones-en-la-educacion_9789264103429-es.

Romero, D., Molina, A. and Chirino, V. (2010). Critical factors in a definition of mobile learning model. In Cruz, M. M. and Moreira, G. (eds.), Handbook of Research on Mobility and Computing, Evolving Technologies and Ubiquitous Impacts. Portugal: IGI Global. Retrieved from http://rita.det.uvigo.es/201011/uploads/IEEE-RITA.2010.V5.N4.pdf.

Soler Fernández, E. (2014). Constructivismo, innovación y enseñanza afectiva. Venezuela: Editorial Equinoccio, Universidad Simón Bolívar. Recuperado de https://books.google.es/books?id=m271PqMmswC\&printsec $=$ frontcover $\&$ hl=es\&source $=$ gbs_vpt_read\#v=onepage \&q\&f=false.

Strauss, A. y Corbin, J. (2002). Bases de la Investigación cualitativa. Técnicas y procedimientos para desarrollar la teoría fundamentada. Antioquia, Colombia: Universidad de Antioquia. Recuperado de https://diversidadlocal.files.wordpress.com/2012/09/bases-investigacion-cualitativa.pdf. 